\title{
Agar culture and chromosome analysis of eosinophilic leukaemia
}

\author{
JOHN M. GOLDMAN 1 , VESNA NAJFELD, AND K. H. TH'NG \\ From the MRC Leukaemia Unit, Hammersmith Hospital, London W12 0HS and the Department of \\ Cytogenetics and Immunology, The Royal Marsden Hospital and the Institute of Cancer Research, \\ London SW3 6JJ
}

SYNOPSIS The blood and bone marrow of a 75-year-old woman with eosinophilia were studied in an attempt to characterize further the entity eosinophilic leukaemia (EL). Haemopoietic cell culture in $\vec{N}$ agar gave patterns of colony size and number and cell morphology indistinguishable from those seen $\mathcal{E}$ in classical chronic granulocytic leukaemia (CGL). Analysis of myeloid cells showed an extra $\mathrm{C}_{\mathrm{O}}$ group chromosome that proved on Giemsa banding to be number 10. These results suggest that some cases of EL represent a chronic myeloproliferative disease that resembles classical CGL but $\vec{\square}$ can be distinguished from it by chromosomal analysis.

When all recognized causes for peripheral blood eosinophilia have been excluded, there remains a small group of patients whose disease appears to be idiopathic. In view of the frequent involvement of heart and lungs, organs not primarily involved in acute or chronic leukaemia, some writers have proposed that this group of patients should be regarded as having a non-neoplastic 'hypereosinophilic syndrome' (Hardy and Anderson, 1968; Chusid et al, 1975). The more popular view is that some at least of these cases are examples of eosinophilic leukaemia (EL), and the controversy has been well reviewed by Benvenisti and Ultmann (1969) and more recently by Zucker-Franklin (1974).

We report here the results of haemopoietic cell culture and chromosome analysis in a 75-year-old woman with hepatosplenomegaly and a greatly raised peripheral blood eosinophil count. Immature cells of the granulocyte series were present in the peripheral blood and bone marrow. Blood and bone marrow culture in agar gave a pattern characteristic of chronic granulocytic leukaemia (CGL) but chromosome analysis showed the presence of an extra $\mathbf{C}$ group chromosome confined to cells of the myeloid series. We believe that the techniques of haemopoietic cell culture in agar and karyotypic

${ }^{1}$ Correspondence and reprint requests to: Dr J. M. Goldman, MRC Leukaemia Unit, Hammersmith Hospital, Ducane Road, London W12 0HS.

Received for publication 26 June 1975. analysis are the most useful approaches to the $\vec{\theta}$ differentation of EL from other non-neoplastic coo of ditions and from other types of leukaemia.

\section{Case report}

A 75-year-old woman was first seen at the Hammersmith Hospital in January 1975. She had suffered from maturity-onset diabetes mellitus for 10 years for which she was taking chlorpropamide and phenformin. Over the previous two weeks she had noticed increasing dyspnoea on exertion and chest pain not clearly related to effort. There was no history of allergy or parasitic disease. Physical examination 0 revealed a pale obese woman in mild congestive 3 . cardiac failure. The pulse was $96 / \mathrm{min}$ and blood $\delta$ pressure $120 / 80 \mathrm{mmHg}$. The jugular venous pressure was raised $5 \mathrm{~cm}$. A third heart sound was audible at 0 the left sternal edge. There were basal crepitations in $>$ both lung fields. The liver and spleen were enlarged to 4 and $10 \mathrm{~cm}$ below the respective costal margins. On investigation the haemoglobin was $10.7 \mathrm{~g} / \mathrm{dl}$, N white cells $80.0 \times 10^{9} / 1$ and platelets $117.0 \times 10^{9} / 1$. N The differential count showed blasts $2 \%$, promyelocytes $1 \%$, myelocytes $6 \%$, neutrophils $20 \%$, eosinophils $43 \%\left(34.0 \times 10^{9} / 1\right)$, basophils $6 \%$, monocytes


showed hypersegmentation, cytoplasmic vacuola- $\stackrel{\infty}{+}$ tion, and occasional basophil granules. The neutro- $\square$ phil alkaline phosphatase score was 5 (normal 40 to

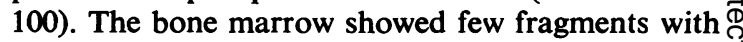


$36 \%$ mature eosinophils and $4.5 \%$ blast cells. Urinalysis and stool examination were negative. The blood sugar was $100 \mathrm{mg} / 100 \mathrm{ml}$. Urea, electrolytes, and other serum chemistry were normal. The electrocardiogram showed normal sinus rhythm with low voltage. The chest radiograph showed moderate enlargement of the heart.

The patient was treated with digoxin and diuretics. Three units of blood were transfused. Her symptoms improved rapidly. She was given busulphan, $50 \mathrm{mg}$ by mouth in a single dose, and discharged from hospital. A moderate reduction in total leucocyte and eosinophil counts was observed but there was no change in the size of the spleen or liver. She was given a further single dose of busulphan, $50 \mathrm{mg}$, eight weeks later. Thereafter the total leucocyte, eosinophil, and blast cell counts began to rise rapidly. There was a transient haematological response to 6-thioguanine, $200 \mathrm{mg}$ daily, for 13 days but by 18 June the total leucocyte count was $300.0 \times$ $10^{9} / 1$ with blasts $3 \%$, neutrophils $3 \%$, and eosinophils $80 \%$. She died the following day. No necropsy was performed.

\section{Methods}

\section{CULTURE OF PERIPHERAL BL.OOD AND}

\section{BONE MARROW in vitro}

Peripheral blood leucocytes and bone marrow nucleated cells were cultured in vitro by the twolayer agar technique and in liquid culture. Both techniques have been described in detail elsewhere (Pike and Robinson, 1970; Goldman et al, 1974; Golde and Cline, 1973; Golde et al, 1974). For agar culture the patient's peripheral blood and bone marrow cells were plated in $0.3 \%$ agar with McCoy's $5 \mathrm{~A}$ medium and fetal calf serum at concentrations of 2,4 , and $8 \times 10^{4} / \mathrm{ml}$ over feeder layers consisting of $1 \times 10^{6}$ normal human peripheral blood leucocytes in $0.5 \%$ agar with McCoy's and fetal calf serum. Cultures were incubated in a humidified atmosphere of $7 \cdot 5 \% \mathrm{CO}_{2}$ and colonies were counted after 10-12 days. Colonies contained more than 50 cells and numbers were standardized and expressed per $1 \times 10^{6}$ nucleated cells. The cellular composition of the colonies was examined at intervals after plating in May-Grünwald-Giemsa stained films. The capacity of the patient's blood to produce CSF was assayed separately by plating $1 \times 10^{6}$ peripheral blood leucocytes as feeder layer under normal human bone marrow cells. In the liquid culture experiments the total viable cell count was assessed by trypan blue dye exclusion, and the cytological composition of the culture was examined in stained preparations at intervals after establishment. Liquid cultures were routinely harvested after 21 days.
CHROMOSOME ANALYSIS

Bone marrow cells were processed by a direct method. After hypotonic treatment in $0.075 \mathrm{M} \mathrm{KCl}$, the material was fixed in methanol and acetic acid (3:1). Peripheral blood cultures were set up in the usual way (Lawler, 1971) using a buffy coat suspension. The cells were maintained for 24,72 , and 120 hours before harvesting.

Giemsa banding patterns of the chromosomes were obtained using the 'ASG' technique (Sumner et al, 1971) slightly modified for use with myeloid cells (Reeves et al, 1972).

\section{Results}

\section{AGAR CULTURE}

There was a good linear relationship between colony numbers counted at the three concentrations of cells plated. In one experiment peripheral blood colony numbers were $410 \pm 68$ (SD), $205 \pm 23$, and $112 \pm$ 15 at concentrations of 8,4 , and $2 \times 10^{4}$ nucleated cells respectively. The results of peripheral blood and bone marrow culture are shown in table $I$ in comparison with a group of patients with $\mathrm{Ph}^{1}$ positive CGL and normal subjects. Mature eosinophils could be identified in colonies after 10 days' culture in both the patient studied and patients with CGL, but in all cases neutrophil polymorphs outnumbered eosinophils. Colony growth was also observed in control plates from which feeder layers had been excluded. Such colonies were generally smaller, more diffuse, and fewer in number than in corresponding plates with normal feeder layers. When the patient's peripheral blood leucocytes were used as feeder layers under normal human bone marrow, the number of colonies cultured after incubation for 12 days was normal.

\section{LIQUID CULTURE}

Mature eosinophils constituted $4 \%$ of cells at day 10 , which compared with $6 \%$ in a patient with $\mathrm{Ph}^{1}+$ ve CGL and $8 \%$ in a control bone marrow obtained from a patient with stage II Hodgkin's disease. Cell viability in the patient under study and CGL patient remained high (table II).

\section{CYTOGENETIC ANALYSIS}

Chromosomes were studied in the bone marrow aspirate, in short-term unstimulated and stimulated peripheral blood cultures, and in peripheral blood leucocytes harvested after 12 days' growth in liquid culture. The results are summarized in table III. The presence of a hyperdiploid clone was detected in direct bone marrow preparations, in unstimulated blood culture harvested after 24 hours, and in leucocytes obtained from long-term liquid culture. 


\begin{tabular}{|c|c|c|c|c|c|c|c|}
\hline & \multirow[t]{2}{*}{ Material } & \multirow{2}{*}{$\begin{array}{l}\text { Total } \\
\text { Leucocytes } \\
\times 10^{9} / l\end{array}$} & \multicolumn{3}{|c|}{ Leucocyte Differential } & \multirow{2}{*}{$\begin{array}{l}\text { Colony Numbers } 1 \\
\text { per } 1 \times 10^{6} \text { Cells }\end{array}$} & \multirow[t]{2}{*}{ Colony Cell Morphology } \\
\hline & & & Blasts & $\begin{array}{l}\text { Eosinophils } \\
\left(\times 10^{9} / l\right)\end{array}$ & Monocytes & & \\
\hline \multicolumn{8}{|l|}{ This patient } \\
\hline 7 Feb. 1975 & PB & $89 \cdot 6$ & $0.9(1 \%)$ & $43.0(48 \%)$ & $5 \cdot 4(6 \%)$ & $4305 \pm 488$ & \\
\hline 28 Feb. 1975 & PB & $51 \cdot 0$ & $1.0(2 \%)$ & $22.4(44 \%)$ & $4 \cdot 1(8 \%)$ & $5215 \pm 850$ & $15 \%$ polymorphs at day \\
\hline 21 March 1975 & PB & $84 \cdot 1$ & $1.7(2 \%)$ & $41 \cdot 2(49 \%)$ & $7.6(9 \%)$ & $3000=1060$ & 10 (neutrophils $10 \%$ \\
\hline 21 March 1975 & $\mathbf{B M}$ & - & $(4.5 \%)$ & $(45 \%)^{2}$ & - & $2562 \pm 936$ & eosinophils $5 \%$ ) \\
\hline \multicolumn{8}{|c|}{$\operatorname{CGL}\left(\mathrm{Ph}^{1}+\mathrm{ve}, \text { untreated }\right)^{3}$} \\
\hline Mean of 13 patients & PB & $212 \cdot 2$ & $9 \cdot 0$ & - & $2 \cdot 0$ & $1350 \pm 1262$ & Neutrophil polymorphs \\
\hline Mean of 8 patients & $\mathbf{B M}$ & $224 \cdot 0$ & - & - & - & $941 \pm 765$ & $\begin{array}{l}\text { usually more numerous } \\
\text { than eosinophils }\end{array}$ \\
\hline \multicolumn{8}{|l|}{ Normal subjects ${ }^{3}$} \\
\hline Mean of 6 donors & PB & - & - & - & - & $10 \pm 6\}$ & see text \\
\hline Mean of 21 donors & $\mathbf{B M}$ & - & - & - & - & $350=124\}$ & \\
\hline
\end{tabular}

Table I Peripheral blood values and agar culture results from blood and bone marrow in the patient with EL compared with those in CGL and normal subjects

${ }^{1}$ Colony numbers are mean values at a plating concentration of $8 \times 10^{4}$ cells $/ \mathrm{ml}$. Results are \pm standard deviation.

'Includes eosinophils at all stages of maturation: $36 \%$ of BM cells were mature eosinophils.

${ }^{3}$ Values for CGL patients taken from Goldman et al (1974); values for peripheral blood of normal donors taken from Chervenick and Boggs,

(1971); values for bone marrow of normal donors are unpublished personal observations.

\begin{tabular}{llll}
\hline & \multicolumn{3}{l}{ Viable Cells $(\%)$} \\
\cline { 2 - 4 } Duration of Culture & $\begin{array}{l}\text { This patient } \\
(P B)\end{array}$ & $\begin{array}{l}P h^{1}+v e \\
C G L \\
(P B)\end{array}$ & $\begin{array}{l}\text { 'Normal' } \\
\text { Control } \\
(B M)\end{array}$ \\
\hline Day 6 (or 7) & 96 & 89 & $72 \cdot 5$ \\
Day 21 & 60 & 60 & 42 \\
\hline
\end{tabular}

Table II Viable cell counts after varying times in liquid culture

$P B=$ peripheral blood; $B M=$ bone marrow.

\begin{tabular}{|c|c|c|c|}
\hline \multirow[t]{2}{*}{ Tissue } & \multirow{2}{*}{$\begin{array}{l}\text { Number of } \\
\text { Cells } \\
\text { analysed }\end{array}$} & \multicolumn{2}{|c|}{ Chromosome Counts } \\
\hline & & 46, $X X$ & $47, x X+10$ \\
\hline BM-direct & 11 & 2 & 9 \\
\hline PB-24 hr & 10 & - & 10 \\
\hline PB-12 days in liquid & & & \\
\hline culture & $24^{1}$ & 4 & 12 \\
\hline PB-72 hr with PHA & 15 & 15 & - \\
\hline PB- $120 \mathrm{hr}$ with PHA & 10 & 10 & - \\
\hline
\end{tabular}

Table III Summary of cytogenetic findings

${ }^{1}$ Eight cells were polyploids showing chromosome lesions associated with long-term culture.

$\mathbf{B M}=$ bone marrow $; \mathbf{P B}=$ peripheral blood $\mathbf{P H A}=$ phytohaemagglutinin.

All hyperdiploid cells had a modal chromosome number of $\mathbf{4 7}$ and showed an extra $\mathrm{C}$ group chromosome. This extra chromosome was identified by Giemsa banding as number 10 (figure). Occasional cells in the bone marrow ( 2 out of 11) and in the longterm liquid cultures ( 4 out of 24 ) had normal diploid karyotypes 46, XX. Peripheral blood lymphocytes had a normal diploid complement.

\section{Discussion}

A persistently raised peripheral blood eosinophil count without demonstrable cause suggests a diagnosis of EL (Zucker-Franklin, 1974; Rickles ang $\vec{c}$ Miller, 1972). The presence in the blood of immature ov eosinophil and neutrophil granulocytes and the presence of specific morphological abnormalities in the mature eosinophils have been taken as further evidence that such patients have a leukaemic disorder (Benvenisti and Ultmann, 1969) but the latter alone are often found in eosinophilia of nonleukaemic origin (Yam et al, 1972; Moore et al, 1973). Though the disease not infrequently terminates in an acute 'blast-cell' phase, its relatively aggressive clinical course from onset, its poor response to busulphan, and the regular absence of the $\mathrm{Ph}^{1}$ chromosome clearly separate it from classical CGL. On the other hand, the percentage of immature cells of the granulocytic series present in the peripheral blood and bone marrow is usually too low to justify inclusion in the category of acute myeloid leukaemia (AML). For the present it seems reasonable to continue to designate these patients, and the one described here, simply as eosinophilic leukaemia.

Analysis of the chromosomes in this patient revealed a clone of cells with an extra number 10 chromosome in direct preparations of bone marrow cells, in unstimulated cultures of peripheral blood (in which immature myeloid cells were present), and in peripheral blood cells after 12 days in liquid culture. The abnormality was absent from peripheral blood lymphocytes stimulated by phytohaemagglutinin and may therefore be regarded as an acquired chromosomal abnormality confined to cells 




Figure Giemsa banding pattern of partial karyotype showing trisomy 10 in a bone marrow cell $(B M)$ and in a peripheral blood cell after 12 days in liquid culture $(P B-12 d)$.

of the myeloid series. The finding of a few cells with a diploid karyotype in bone marrow preparations ( 2 out of 11$)$ and in long-term liquid culture ( 4 out of 24) reflects the proliferative advantage of the clone of cells with the acquired trisomy 10.

Aneuploidy involving one or other $\mathrm{C}$ group chromosome is a common abnormality in bone marrow cells of patients with a variety of chronic myeloproliferative or maturational disorders. An extra number 8 chromosome has been identified in patients with thrombocytopenia, with pancytopenia, and with sideroblastic anaemia (De La Chapelle et al, 1972; Jonasson et al, 1974). An extra number 9 chromosome was identified as the sole chromosomal aberration in myelosclerosis (Davidson and Knight, 1973) and in association with other chromosomal abnormalities in patients with polycythaemia and thrombocytosis (Rowley, 1973a).

In addition to the presence of the $\mathrm{Ph}^{1}$ chromosome there are occasional reports of chromosomal aberrations involving an extra $\mathrm{C}$ group chromosome in bone marrow cells of patients in the chronic phase of CGL (Goh et al, 1964; Tough, 1965; de Grouchy et al, 1966; Whang-Peng et al, 1968). Moreover, chromosome gain in the $\mathrm{C}$ group frequently occurs in patients with $\mathrm{Ph}^{1}$-positive $\mathrm{CGL}$ in association with acute 'blast-cell' transformation; acquired trisomy in this situation has so far been identified in chromosome numbers 7 (Kaffe et al, 1974), 8 (Rowley, 1973b), 10 (Massachusetts General Hospital, 1973), and 12 (Kaffe et al, 1974). Thus the only example of trisomy 10 so far described in chronic myeloproliferative disorders was found in a
$\mathrm{Ph}^{1}$-positive cell population. When one considers that patients with $\mathrm{Ph}^{1}$-negative CGL usually have a normal karyotype (Sandberg and Hossfeld, 1970; Mitelman et al, 1974) the finding of an acquired trisomy for chromosome 10 suggests that this case of EL should be regarded as a chronic myeloproliferative disorder that appears cytogenetically distinct from both $\mathrm{Ph}^{1}$-positive and $\mathrm{Ph}^{1}$-negative CGL.

In $\mathrm{Ph}^{1}$-positive CGL the number of colony- and cluster-forming cells in the blood is far higher than normal and there is a corresponding though less impressive increase observed in cultures of bone marrow cells (Goldman et al, 1974; Moore et al, 1973; Moberg et al, 1974). Similar increases in circulating and bone marrow colony- and clusterforming cell numbers have also been reported in three patients with $\mathrm{Ph}^{1}$-negative CGL (Moore, 1974). Colony formation remains dependent on an exogenous source of CSF without which the colonies cultured are few and small. In the patient reported here agar culture of blood leucocytes in the presence of CSF gave colony numbers higher than the mean value but well within the range of that seen in CGL. The size of the colonies was also typical of those seen in CGL. In the bone marrow cultures colony numbers were three times higher than the mean values for CGL, but this discrepancy might to some extent have been accounted for by contamination of the patient's bone marrow aspirate with peripheral blood which can spuriously increase colony numbers. Thus the number of colony-forming cells (CFUc) in the peripheral blood in CGL and in this case of EL is in general 100 to 500 times higher than in normal sub- 
jects; in the bone marrow CFUc numbers are increased three or more times in comparison with normal.

Though CSF has not so far been shown to play a role in the maintenance of granulocyte or monocyte homeostasis in vivo, its principal source in the agar culture system is the peripheral blood monocyte. In CGL, monocyte numbers are reduced in comparison with the greatly increased granulocyte counts, and the peripheral blood leucocytes are a poor source of CSF when used in conventional concentrations (Goldman et al, 1974). In this patient the percentage of monocytes in the peripheral blood was normal, and peripheral blood CSF production was accordingly maintained. This difference between this patient and classical CGL would also explain our finding of spontaneous colony formation in the absence of feeder layers.

Eosinophil precursors grow well in the agar system, and eosinophils may predominate in colonies grown from the blood of normal persons (Chervenick and Boggs, 1971). In colonies cultured from CGL klood and bone marrow eosinophils are also seen but are generally inconspicuous, and in this patient the number of mature neutrophils in colonies examined after 10 days in culture always exceeded the number of mature eosinophils. It is of note that eosinophils were absent from the colonies grown from the blood of a previously reported patient thought to have an eosinophilic variant of AML (Paran et al, 1970). Eosinophils need not therefore predominate in colonies cultured from patients with EL.

We do not telieve that the agar colony pattern sean in this patient is typical of all cases of EL. Preliminary results in two further cases studied very recently suggest that peripheral blood CFUc numbers are not always raised, but at least one of these patients was regarded as an eosinophilic variant of AML. It seems likely that just as EL may stand at the 'leukaemic' end of the hypereosinophilic spectrum, so EL may itself comprise a continuum in which some cases are eosinophilic variants of AML and others are forms of a chronic mycloproliferative disease. The patient described in this report comes into the latter category.

The findings of a specific chromosomal abnormality in this patient's myeloid cells and the failure to demonstrate a predominance of mature eosinophils in culture suggest that the granulocytic stem cell is involved in the leukaemic process. Why the manifestations of this process should primatily involve cells of the eosinophilic series in vivo is not clear. Nevertheless we conclude that the techniques of chromosome analysis and in vitro haemopoietic cell culture are among the most useful for the characterization of EL.
The authors are grateful to Professor D. A. G. Galton for helpful advice during the preparation of $\underline{\underline{0}}$ this manuscript. V. N. is in receipt of a Ph.D. grant from the Royal Marsden Hospital and Institute of $\stackrel{\vec{P}}{+}$ Cancer Research, London and is particularly indebted to Dr Sylvia D. Lawler for continued interest and help.

\section{References}

Benvenisti, D. S. and Ultmann, J. E. (1969). Eosinophilic leukemia : report of five cases and review of literature. Ann. intern. Med., 71, 731-745.

Chervenick, P. A. and Boggs, D. R. (1971). In vitro growth of granulocytic and mononuclear colonies from blood of normal individuals. Blood, 37, 131-135.

Chusid, J. M., Dale, D. C., West, B. C., and Wolff, S. M. N (1975). The hypereosinophilic syndrome: analysis of fourteen cases with review of the literature. Medicine ? (Baltimore), 54, 1-27.

Davidson, W. M. and Knight, L. A. (1973). Acquired trisomy 9. (Letter). Lancet, 1, 1510 .

De La Chapelle, A., Schröder, J., and Vuopio, P. (1972). 8-Trisomy in the bone marrow: report of two cases. Clin. Genet., 3, 470-476.

Goh, K. O., Swisher, S. N., and Troup, S. B. (1964). Submetacentric chromosome in chronic myelocytic leukemia. Arch. intern. Med., 114, 439-443.

Golde, D. W., Byers, L. A., and Cline, M. J. (1974). Chronic myelogenous leukemia cell growth and maturation liquid culture. Cancer Res., 34, 419-423.

Golde, D. W. and Cline, M. J. (1973). Growth of human bo marrow in liquid culture. Blood, 41, 45-57.

Goldman, J. M., Th'ng, H. K., and Lowenthal, R. M. (1974 In vitro colony forming cells and colony stimulating factor in chronic granulocytic leukaemia. Brit.J. Cancer, 30, 1-12.

de Grouchy, J., de Nava, C., Cantu, J. M., Bilski-Pasquier, G., and Bousser, J. (1966). Models for clonal evolution: a study of chronic myelogenous leukemia. Amer. J. hum. Genet., 18, 485-503.

Hardy, W. R. and Anderson, R. E. (1968). The hypereosinophilic syndrome. Ann. intern. Med., 68, 1220-1229.

Jonasson, J., Gahrton, G., Lindsten, J., SimonssonLindemalm, C., and Zech, L. (1974). Trisomy 8 in acute myeloblastic leukemia and sideroachrestic anemia. Blood, 43, 557-563.

Kaffe, S., Hsu, L. Y. F., and Hirschhorn, K. (1974). Acquired trisomies 12 and 7. (Letter). Lancet, 1, 261-262.

Lawler, S. D. (1971). Techniques for chromosomal analysis. Broadsheet No. 74 (June 1971), pp. 1-7. Association of Clinical Pathologists, London.

Massachusetts General Hospital (1973). Case records: case 18-1973. New Engl. J. Med., 288, 957-963.

Mitelman, F., Brandt, L., and Nilsson, P. G. (1974). The banding pattern in Philadelphia chromosome negative chronic myeloid leukaemia. Hereditas (Lund.), 78, 302-304. ज

Moberg, D., Olofsson, T., and Olsson, I. (1974). Granulopoiesis in chronic myeloid leukaemia. I. In vitro cloning of blood and bone marrow cells in agar culture. Scand. J. N Haemat., 12, 381-390.

Moore, M. A. S. (1974). In Advances in Acute Lcukaemia, edited by F. J. Cleton, D. Crowther, and J. S. Malpas, p. 161. North Holland Publishing Company, Oxford.

Moore, M. A. S., Williams, N., and Metcalf, D. (1973). 온 In vitro colony formation by normal and leukemic human hematopoietic cells: characterization of the colony-forming cells. J. nat. Cancer Inst., 50, 603-623.

Paran, M., Sachs, L., Barak, Y., and Resnitsky, P. (1970). 
In vitro induction of granulocyte differentiation in hemopoietic cells from leukemic and non-leukemic patients. Proc. nat. Acad. Sci. (Wash.), 67, 1542-1549.

Pike, B. L. and Robinson, W. A. (1970). Human bone marrow colony growth in agar-gel. J. Cell Comp. Physiol., 76, 77-84.

Reeves, B. R., Lobb, D. S., and Lawler, S. D. (1972). Identity of the abnormal F-group chromosome associated with polycythaemia vera. Humangenetik, 14, 159-161.

Rickles, F. R.. and Miller, D. R. (1972). Eosinophilic leukemoid reaction. J. Pediat., 80, 418-428.

Rowley, J. D. (1973a). Acquired trisomy 9. (Letter). Lancet, 2, 390.

Rowley, J. D. (1973b). A new consistent chromosomal abnormality in CML identified by quinacrine fluorescence and Giemsa staining. Nature (Lond.), 243, 290-293.

Sandberg, A. A. and Hossfeld, D. K. (1970). Chromosomal abnormalities in human neoplasia. Ann. Rev. Med., 21, 379-408.

Sumner, A. T., Evans, H. J., and Buckland, R. A. (1971). New technique for distinguishing between human chromosomes. Nature [New Biol.], 232, 31-32.

Tough, I. M. (1965). Cytogenetic studies in cases of chronic myeloid leukaemia with a previous history of radiation. In Current Research in Leukaemias, edited by F. G. J. Hayhoe, pp. 47-54. Cambridge University Press, London.

Whang-Peng, J., Canellos, G. P., Carbone, P. P., and Tjio, J. H. (1968). Clinical implications of cytogenetic variants of chronic myelocytic leukemia (CML). Blood, 32, 755-766.

Yam, L. T., Li, C. Y., Necheles, T. F., and Katayama, I. (1972). Pseudoeosinophilia, eosinophilic endocarditis and eosinophilic leukemia. Amer. J. Med., 53, 193-202.

Zucker-Franklin, D. (1974). Eosinophil function and disorders. Advanc. intern. Med., 19, 1-25. 